\title{
DOLARIZACIÓN DE LOS COSTOS agrícolas EN Argentina
}

\begin{abstract}
Pablo Wahren ${ }^{\mathrm{a}}$
Fecha de recepción: 27 de enero de 2020. Fecha de aceptación: 8 de julio de 2020 .

$$
\text { http://doi.org/10.22201/iiec.20078951e.2020.203.69560 }
$$

Resumen. El presente artículo estudia los determinantes de los costos agrícolas en Argentina y en particular su grado de dolarización. Para estimar la dolarización de los costos se construyó una base de costos agrícolas por región para los tres principales cultivos del país y se aplicó econometría de panel. Se argumenta que es relevante incorporar esta dimensión de análisis para el diseño de esquemas de devaluaciones compensadas, ya que un alto grado de dolarización reduce su eficacia como mecanismo de desacople de precios sin efectos distributivos. La presente investigación aporta evidencias empíricas para repensar las recomendaciones tradicionales sobre políticas económicas propias de la estructura productiva argentina a partir de las transformaciones tecnológicas y organizacionales del sector agrícola.
\end{abstract}

Palabras clave: costos agrícolas; dolarización; cultivos agrícolas; política agrícola; análisis econométrico.

Clasificación JEL: C23; N56; O13; Q11; Q17.

\section{Dollarization of Agricultural Costs in Argentina}

\begin{abstract}
This article analyzes the determinants of agricultural costs in Argentina, giving special attention to their degree of dollarization. To estimate the dollarization of agricultural costs, an agricultural cost base by region was calculated for the country's three primary crops, to which panel econometrics were then applied. The present article argues that incorporating this dimension of analysis is vital when designing compensated devaluation schemes, given that a high degree of dollarization reduces such schemes' effectiveness as a mechanism for decoupling prices without distributive effects. This research contributes empirical evidence drawn from the technological and organizational transformations in the agricultural sector with which to rethink the traditional recommendations of the economic policies of Argentina's productive structure.
\end{abstract}

Key Words: agricultural costs; dollarization; agricultural crops; agricultural policy; econometric analysis.

\footnotetext{
${ }^{a}$ Centro de Innovación para los Trabajadores (CITRA)/Consejo Nacional de Investigaciones Científicas (CONICET), Argentina. Correo electrónico: pwahren@conicet.gov.ar
} 


\section{INTRODUCCIÓN}

En los últimos 40 ańos el sector agrícola argentino ha experimentado enormes cambios a nivel tecnológico y organizacional. Como resultado, la producción se multiplicó por tres, el área sembrada se duplicó y la soja pasó de ser un cultivo marginal a representar más de la mitad de la producción agrícola. Tales transformaciones han llevado a diversos autores a repensar esta actividad y a considerar la existencia de un nuevo agro argentino (Teubal, 2001; Trigo, 2005; Bisang et al., 2008; Gras y Hernández, 2016).

La agricultura no está exenta del proceso de globalización registrado en la industria desde la década de los sesenta del siglo xx. En efecto, en el ámbito académico existe cierto consenso en que, al igual que en el sector industrial, el sector agrario pasó a organizarse en una lógica globalizada de cadena, con preeminencia de empresas multinacionales en los eslabones claves. Un aspecto en el que coinciden estas miradas es que las empresas transnacionales ligadas al desarrollo químico y biotecnológico comandan el proceso de innovación, siendo responsables y difusoras de las principales transformaciones tecnológicas y de cambios en los marcos regulatorios para aprobar el uso de nuevas tecnologías (Teubal, 2001; Pengue, 2005; Bisang et al., 2010). Diversos trabajos señalan que el sector pasó a organizarse como una Cadena Global de Valor (CGV) (Humphrey y Memedovic, 2006; Bisang et al., 2010; Lee et al., 2012).

Algunos análisis sobre los cambios en el sector agropecuario se ubicaron en los costos (Bisang et al., 2008; Makler, 2008; Pierri, 2017; CREA, 2018). En esta dirección, Bisang et al. (2008) señalan que con la preeminencia de insumo plenamente transable se asistió a una dolarización ${ }^{1}$ directa de varios insumos claves. En el marco de la volatilidad cambiaria que atraviesa Argentina desde 2018, este aspecto ha adquirido relevancia en el debate público.

Ahora bien, ¿qué magnitud real alcanza la dolarización?, ¿cómo afecta la misma al diseño de política económica?

En particular, si los costos agrícolas se encuentran dolarizados la instrumentación de devaluaciones compensadas debe ser reexaminada. En esta dirección, el presente trabajo analiza si el impacto de esta política, propia de países semi-industrializados y productores de alimentos, ha sido alterado por el cambio tecnológico y la transnacionalización del sector agrícola. En este sentido, el trabajo busca, desde la visión de un país periférico, realizar un aporte a las discusiones académicas recientes que señalan que el impacto de

1 La dolarización es entendida como una baja o nula elasticidad de un costo medido en dólares a variaciones del tipo de cambio. 
las políticas macroeconómicas se ha modificado a partir de la globalización y la organización de la producción en CGV (Kosacoff y López, 2008; Gereffi y Sturgeon, 2013; OECD, 2018).

La investigación presenta un diseño cuantitativo y recurre al análisis de entrevistas semi-estructuradas ${ }^{2}$ para explicar los determinantes de los costos agrícolas y para construir los modelos econométricos con los cuales se estima el impacto del tipo de cambio sobre los costos.

El artículo se estructura en cinco secciones. En la sección 2 se desarrolla el origen y planteo de las devaluaciones compensadas, y se analizan los determinantes de los costos agrícolas. En la sección 3 se explica la metodología y las fuentes de información utilizadas para estimar la dolarización de los costos agrícolas. En la sección 4 se presentan los resultados, en especial las elasticidades de los distintos tipos de costos al tipo de cambio según cultivo. Por último, en la quinta sección se desarrollan las conclusiones y se presentan discusiones de política económica asociadas.

\section{MARCO DE ANÁLISIS}

\section{Debates en torno a la política cambiaria para el desarrollo}

Diversos autores sostienen la conveniencia de aplicar un Tipo de Cambio Real Competitivo y Estable (TCRCE) en economías periféricas semi-industrializadas (Frenkel y Ros, 2006; Bresser-Pereira, 2007; Rodrick, 2007). Existen tres canales principales por los cuales un tipo de cambio real alto y estable es beneficioso para la economía (Frenkel y Ros, 2006):

1) Canal de crecimiento: al reducir los costos en dólares de la producción local, esta política permite ampliar la producción de bienes transables al fomentar su colocación en el mercado interno y externo.

2) Canal de intensidad laboral: se basa en que el TCRCE modifica al alza la elasticidad entre el crecimiento económico y el empleo, al abaratar relativamente la mano de obra por sobre los bienes de capital (en particular los importados). De este modo, la creación de puestos de trabajo derivada del aumento de la actividad económica será potenciada por este efecto.

2 Referente a 12 entrevistas semi-estructuradas durante el periodo transcurrido entre marzo 2017 y marzo 2019. Para mayor información véase Wahren (2019). 
3) Canal de desarrollo: en el largo plazo el TCRCE da lugar a la aparición y la consolidación de nuevas y más complejas actividades productivas. En esta línea, se destaca que el impulso a la producción de bienes transables tiene un carácter estratégico para los países en desarrollo, en tanto en ese sector se encuentran las mayores posibilidades de learning by doing y de derrames tecnológicos hacia el resto de la economía (Razmi et al., 2012).

Sin embargo, la estrategia de TCRCE para alcanzar el desarrollo económico ha recibido diversas críticas desde la perspectiva de una economía semi-industrializada especializada en la producción agraria. Las críticas apuntan al no cumplimiento de las relaciones planteadas y a los efectos regresivos en materia de distribución del ingreso que esta política conlleva.

El primer grupo de críticas se orienta hacia el canal del crecimiento. En línea con los argumentos de la devaluación contractiva (Diaz Alejando, 1963), se señala que en el corto plazo si los movimientos depreciatorios para alcanzar tipo de cambio competitivo generan alzas de precios que derivan en reducciones de salario real, el resultado será un menor nivel de actividad económica. En el mediano plazo, si la demanda doméstica no se recupera, a causa de la nueva distribución del ingreso más regresiva, el efecto sobre el crecimiento económico de los nuevos precios relativos se puede anular por completo (Fiorito et al., 2015; López, 2008).

Por otro lado, el impacto en las exportaciones podría ser limitado. Los únicos productos que bajo los nuevos precios relativos se pueden vender al exterior son los que se encontraban cerca de un piso de rentabilidad mínima y cuya competencia en el mercado mundial se da vía precios (Crespo y Lazzarini, 2012). Asimismo, un incremento de las exportaciones de un país, debido a una devaluación, implicaría una menor cuota de mercado para otros países exportadores, que podrían usar la misma estrategia devaluatoria para recomponer la situación (Dvoskin y Feldman, 2015).

En cuanto al canal empleo, no necesariamente un incremento del tipo de cambio aumenta la intensidad laboral. No existe una causalidad inversa entre el precio de los factores y su demanda. Para producir una determinada mercancía se utilizan distintos tipos de bienes de capital, por lo que no se puede considerar al capital de manera homogénea. El problema entonces es que no podrá incrementarse la producción vía mayor intensidad laboral si no se modifica la forma física del acervo de bienes de capital. En este sentido es posible que una verdadera sustitución de capital por trabajo sólo pueda ser rebajando el salario real a un mínimo por debajo de la subsistencia (Fiorito et al., 2015; Dvoskin y Feldman, 2015). 
La tercera crítica apunta a la sostenibilidad de este esquema. En línea con el análisis de Diamand (1972), las devaluaciones al reducir el poder adquisitivo del salario derivan en una puja distributiva donde los trabajadores intentan recuperar lo perdido. De esta manera se generaliza una espiral de precios y salarios que eleva el valor de los bienes no-transables al de los transables (Kicillof y Nahón, 2007; Dvoskin y Feldman, 2015).

El conjunto de críticas desarrolladas apunta a entender por qué en economías basadas en la exportación de alimentos como Argentina podrían no cumplirse las relaciones planteadas por el primer conjunto de autores. Frente a estas particularidades de la estructura productiva argentina, Diamand (1972) encuentra un posible camino alternativo el programa de estabilización de 1967, al cual cataloga como una devaluación compensada. Este programa implicó conjuntamente la devaluación del tipo de cambio nominal con un incremento de los derechos de exportación. El resultado fue un incremento del tipo de cambio real para la industria, mientras que el del agro se mantuvo estable. En líneas generales, se define a una devaluación compensada como aquella que atenúa o anula los efectos inflacionarios y regresivos sobre la distribución del ingreso de una devaluación (López y Pacheco, 2002). Dada la estructura productiva argentina, se lograría principalmente a través de derechos de exportación sobre los productos agrícolas. Por lo tanto, el objetivo de esta medida sería evitar el impacto regresivo, y por tanto recesivo, que resulta del encarecimiento del precio de los alimentos tras una devaluación, mientras se favorece cambiariamente al sector menos competitivo (Abeles y Panigo, 2015).

El presente artículo busca realizar un aporte al diseño de las devaluaciones compensadas para economías semi-industrializadas con preeminencia agraria. De esta forma, argumenta que los cambios en la estructura de las cadenas de valor agrícolas imponen nuevos desafíos que deben ser incorporados a la teoría. Esto se debe a que si los costos se encuentran total o parcialmente dolarizados, la aplicación de un derecho de exportación de la magnitud necesaria para evitar el traslado a precios locales de la devaluación captaría parte del excedente agrícola existente previamente, dado el incremento de costos -en moneda doméstica- resultante. Este fenómeno reviste implicaciones políticas que pueden condicionar o alterar la eficacia de las devaluaciones compensadas.

\section{La determinación de los costos agrícolas}

El origen de que los costos agrícolas se encuentran dolarizados es político y se puede rastrear en 2002, cuando entidades agrarias reclamaban que "la devaluación [de ese año] no había beneficiado al sector agropecuario; por el 
contrario no sólo no mejoraba la competitividad del país, sino que además incrementaba los costos de los insumos y deterioraba los precios relativos" (Makler, 2008, p. 10). En relación con dicho episodio devaluatorio, Pierri (2017) señala que los costos de producción en moneda doméstica se incrementaron $181 \%$ entre 2001 y 2002, muy por encima de la suba del índice de precios al consumidor (29.5\%). Concluye que el extraordinario aumento del ingreso en moneda doméstica de los productores, derivado de la devaluación, fue atenuado por el incremento de los ingresos de las firmas proveedoras de insumos y los contratistas.

Estos planteamientos se encuentran en línea coincidente con Bisang (2007, p. 220), quien señala que:

La nueva conformación de la estructura productiva, articulada con una mayor preeminencia de "lo industrial" sobre el agro y con la presencia de insumos plenamente transables se produce una suerte de "dolarización" directa de varios de los insumos claves del modelo.

A continuación se presenta una descripción de los determinantes de los principales costos agrícolas y su evolución en el nuevo modelo productivo.

\section{Semillas}

El costo de producción de una semilla fiscalizada acarrea en primer lugar los costos del propio commodity que se busca reproducir. A eso se le suma el costo de embolsado, fiscalización, marketing y el costo indirecto de innovación. Asimismo, cuando el evento transgénico o el germoplasma se encuentra patentado se agrega el pago de regalías por ello. Las firmas locales que participan en el desarrollo de semillas lo hacen a partir del uso de eventos transgénicos patentados -y muchas veces también de germoplasmas-, por los cuales deben pagar regalías a alguna compañía multinacional. En este sentido, un directivo de una importante semillera local planteó que en 2018 para empresas nacionales las regalías por utilizar el evento transgénico y el germoplasma desarrollado en una semilla intacta representó $60 \%$ del costo de producción (entrevista realizada en mayo de 2018). Según un miembro del equipo de investigación de la revista Márgenes Agropecuarios, en el caso de las semillas no fiscalizadas el costo del grano en el mercado se vuelve aún más relevante sobre el total (entrevista realizada en diciembre de 2018). El precio de la semilla no fiscalizada se establece a partir de la suma del precio del grano más el costo de limpieza, el costo del análisis germinativo (costo de laboratorio), el costo de almacenamiento y el costo de embolsado. 
Por lo tanto, los costos dolarizados de las semillas son principalmente dos: el pago de las regalías que son fijadas en dólares, elemento muy relevante en el caso de semillas fiscalizadas de última generación que aún tienen patente vigente. Y el precio del commodity, ya que el grano de la cosecha anterior se convierte en insumo de producción tanto en la semilla fiscalizada como en la no fiscalizada. Habría que agregar que se incrementó el grado de transabilidad de las semillas, en particular en lo que refiere a las de maíz (Ministerio de Agroindustria, 2016).

\section{Agroquímicos y fertilizantes}

Desde la Revolución Verde adquirió relevancia el uso de agroquímicos, los cuales en su mayoría son desarrollados y, al menos inicialmente, producidos por firmas multinacionales para todo el mundo. Cabe destacar que, aunque las patentes de herbicidas y fertilizantes tienen un plazo determinado, constantemente se desarrollan nuevas innovaciones por parte de las grandes marcas con las que sostienen su predominio en el mercado. No obstante, aun cuando las patentes se vencen, los precios tienden a fijarse en términos internacionales. Lo que se vincula con el grado de transabilidad internacional que adquirieron estos productos como resultado del proceso de homogeneización de las técnicas productivas a nivel global. Los principios activos de los principales agroquímicos son importados como producidos localmente y exportados. Tal es el caso de los herbicidas A 2, 4 D, Acetoclor, Atrazina y Glifosato como del insecticida Cipermetrina. Lo mismo ocurre con los fertilizantes de toda clase. Asimismo, entre los insumos básicos para la producción de agroquímicos y fertilizantes destaca el petróleo cuyo precio se fija a nivel internacional. Por último, un elemento central para explicar la evolución del precio de los agroquímicos, que surgió de la entrevista realizada a un investigador del Movimiento CREA, es su relación positiva con el precio de los granos (entrevista realizada en octubre de 2018). Esto se debe a disputas por la apropiación del excedente al interior de la cadena.

\section{Labranza}

Los principales costos por la tarea de labranza son la mano de obra, el gasoil y las amortizaciones de la maquinaria. Se trata de la actividad de trabajo más intensiva y, por tanto, con mayor incidencia de costos en moneda local. No obstante, el pasaje a la siembra directa implica una menor cantidad de trabajos 
por hectárea, ya que elimina la tarea de roturar la tierra y los procesos asociados (Lódola, 2008). Esta dinámica puede haber comprendido una dolarización de los costos al reducir los correspondientes a la mano de obra. Asimismo, al interior de las labores (además de la siembra, incluye cosecha y tareas de cuidado) tendió a reemplazarse trabajo por capital, con lo cual se desplazó un costo típicamente en moneda local (salario), por otro más dolarizado: el costo de maquinarias y sus amortizaciones. Esto último atravesó distintas fases. En un primer momento se destacó la importación de maquinaria importada, pero en los últimos años se asistió a un importante proceso de sustitución de importaciones. No obstante, dado el creciente peso que tienen las exportaciones y los contenidos importados (partes y piezas) de la producción local, los precios tienden a moverse en línea con los internacionales. En línea con la maquinización de las tareas adquirió mayor relevancia el precio del gasoil cuyo grado de transabilidad es elevado. ${ }^{3}$

\section{Cosecha}

La cosecha, pese a usar insumos de producción similares, tiene una lógica distinta en la determinación de su precio. En primer lugar, para la cosecha se necesita una escala mayor, por lo que predomina la modalidad de contratista tal como refleja el Censo Nacional Agropecuario 2018 (INDEC, 2020). En segundo lugar, los contratos se pactan en su mayoría a porcentaje de la cosecha. La generalización de este esquema implicó una importancia creciente del precio internacional en la determinación de este costo, y, por ende, una relación directa con el tipo de cambio. Un investigador de una reconocida asociación de productores destacó que dicho porcentaje suele oscilar entre 5 y $7 \%$ de lo cosechado, fluctuando según las variaciones en el rinde y en los precios (entrevista realizada en diciembre de 2018). El costo de la cosecha, por su parte, puede variar entre zonas según la mayor o menor oferta de equipos disponibles (Vilulla y Chen, 2015).

\section{Comercialización}

Los gastos de comercialización se dividen entre el flete y las tareas de acopio y acondicionamiento de los granos. Según la revista Márgenes Agropecuarios, las

3 Cabe aclarar que el precio del gasoil en Argentina atravesó por años de regulaciones que disociaron el precio local del internacional. 
tareas de acondicionamiento en el caso de la soja son especialmente el secado y zarandeo, en maíz la paritaria y el secado, y en trigo la paritaria. El flete se divide en dos: corto y largo. El primero va del campo al centro de acopio, y el segundo de este último al puerto. El flete representa entre el 60 y $90 \%$ de los gastos comerciales en todos los cultivos y regiones, registrándose su menor incidencia en la soja por ser el cultivo donde se realizan más tareas de acondicionamiento. Asimismo, cabe destacar que cuanto mayor es la cercanía del campo al puerto menor es el costo del flete. Por ejemplo, en 2016 el flete representó $60.8 \%$ del total en el norte de Buenos Aires, y 83\% en Salta, según datos de Márgenes Agropecuarios. El flete se pacta y paga en pesos. En cambio, los gastos de acopio y acondicionamiento conllevan una lógica más dolarizada, ya que se fijan a porcentaje de la cosecha, por lo que se relacionan directamente con los precios internacionales, y por ende están directamente relacionados con el tipo de cambio.

\section{METODOLOGíA}

\section{Base de datos y econometría}

Con el objetivo de cuantificar el grado de dolarización de los costos se diseñó una base de panel que contiene los principales costos en dólares de cada uno de los tres principales cultivos del campo argentino: soja, trigo y maíz. La selección de éstos se debe a que representan $87.1 \%$ de la producción agrícola argentina.

El término "panel de datos" se refiere a la muestra de observaciones crosssection de las variables dependientes (costo de labranza, costo de semillas, costo de agroquímicos, costo de cosecha y gastos de comercialización) e independientes a través del tiempo. La información de costos fue obtenida de Márgenes Agropecuarios, que publica mensualmente datos de costos y márgenes en dólares del sector agropecuario. Para realizar las estimaciones que atañen al objetivo propuesto se utilizaron datos referidos a soja de primera ${ }^{4}$ (en adelante soja), maíz y trigo comprendidos entre enero de 2003 y diciembre de 2016. Si bien los datos son mensuales, se trimestralizaron a fin de reducir volatilidad y aumentar el poder explicativo de los modelos. La selección del periodo se debe a tres factores: 1) analizar un periodo de tiempo donde predominen las técnicas productivas que caracterizan al nuevo agro argentino, 2) en el cual se

4 Cabe señalar que la soja de primera representa aproximadamente $70 \%$ del cultivo total de soja (Bolsa de Cereales, 2018). 
registra volatilidad en el tipo de cambio nominal y real, y 3) que contiene un elevado número de observaciones.

La muestra contiene 168 observaciones mensuales y 56 trimestrales para cinco regiones en el caso del maíz, cuatro en el caso del trigo y tres en el de soja (véase cuadro 1). Por cada cultivo y región se cuenta con dos tipos de rindes distintos. Asimismo, para algunas de las principales regiones se presenta información de más de una técnica. De todas las regiones y técnicas con información disponible se seleccionaron exclusivamente aquellas que presentan datos para la totalidad del periodo de análisis.

Esta base provee las variables a explicar, las cuales son cada tipo de costo (labranza, semillas, agroquímicos, cosecha, comercialización y totales) para cada uno de los tres cultivos seleccionados. Para construir las variables explicativas se utilizaron diversas fuentes públicas y privadas. Los datos de tipo de cambio y salarios provienen de organismos públicos nacionales como el Banco Central de la República Argentina y el Ministerio de Trabajo, respectivamente. Los precios del gasoil provienen de la base de datos de CREA. Los precios internacionales de los distintos commodities se desprenden del Fondo Monetario Internacional (FMI). Los derechos de exportación se tomaron de un relevamiento realizado por la Bolsa de Cereales de Rosario. Dado que Márgenes Agropecuarios presenta los costos, medidos en dólares, a principio de cada mes, para realizar las regresiones se utilizará el valor del tipo de cambio oficial del primer día hábil del mes (al ser el vigente al momento de confección de los costos). ${ }^{5}$ Para las demás variables se tomará el dato del mes anterior, por ser el que influye en la determinación del costo del mes corriente. Por último, se incorpora también una dummy por cultivo para registrar cambios técnicos presentados por la revista y captar las variaciones en los costos derivadas de este efecto. ${ }^{6}$ Estos quiebres se registran en distintos periodos según el cultivo: la soja en octubre de 2006, el maíz en abril de 2012 y el trigo en diciembre de 2011.

En otras palabras, el efecto de las depreciaciones sucedidos en una fecha posterior a la publicación de la revista se puede observar recién al mes siguiente. Cabe agregar que, si bien entre noviembre de 2011 y diciembre de 2015 en Argentina existieron diferentes tipos de cambio, se utilizará el tipo de cambio oficial para todo el periodo dado que el efecto del no oficial sobre la inflación fue pequeño (Frenkel y Fridheim, 2016).

6 Márgenes Agropecuarios presenta los costos asociados a distintas técnicas productivas. El cambio técnico refleja cambios en la composición de los costos asociados a la introducción de nuevos productos tecnológicos que derivan en una nueva combinación de insumos. Al producirse el cambio se registra un quiebre relevante para cada cultivo que será captado por las mencionadas variables dummies. 
Cuadro 1. Series por cultivo, región, técnica y rinde para el periodo 2003-2016

\begin{tabular}{|c|c|c|c|c|c|c|c|}
\hline 10 & Cultivo & Región & Técnica $^{a}$ & Rinde $^{b}$ & Observaciones & $\begin{array}{c}\text { Fecha de } \\
\text { inicio }\end{array}$ & $\begin{array}{c}\text { Fecha de } \\
\text { finalización }\end{array}$ \\
\hline 11 & Maíz & N. Buenos Aires - S. Santa Fe & 1 & 75 & $168 / 56$ & ene-03 & dic-16 \\
\hline 12 & Maíz & N. Buenos Aires - S. Santa Fe & 1 & 95 & $168 / 56$ & ene-03 & $\operatorname{dic}-16$ \\
\hline 21 & Maíz & N. Buenos Aires - S. Santa Fe & 2 & 75 & $168 / 56$ & ene-03 & dic-16 \\
\hline 22 & Maíz & N. Buenos Aires - S. Santa Fe & 2 & 95 & $168 / 56$ & ene-03 & dic-16 \\
\hline 31 & Maíz & N. Buenos Aires - S. Santa Fe & 3 & 75 & $168 / 56$ & ene-03 & dic-16 \\
\hline 32 & Maíz & N. Buenos Aires - S. Santa Fe & 3 & 95 & $168 / 56$ & ene-03 & dic-16 \\
\hline 41 & Maíz & 0. Buenos Aires & 4 & 65 & $168 / 56$ & ene-03 & $\operatorname{dic}-16$ \\
\hline 42 & Maíz & 0. Buenos Aires & 4 & 85 & $168 / 56$ & ene-03 & $\operatorname{dic}-16$ \\
\hline 51 & Maíz & S. Entre Ríos & 5 & 65 & $168 / 56$ & ene-03 & $\operatorname{dic}-16$ \\
\hline 52 & Maíz & S. Entre Ríos & 5 & 85 & $168 / 56$ & ene-03 & $\operatorname{dic}-16$ \\
\hline 61 & Maíz & SE. Buenos Aires & 6 & 70 & $168 / 56$ & ene-03 & dic-16 \\
\hline 62 & Maíz & SE. Buenos Aires & 6 & 90 & $168 / 56$ & ene-03 & dic-16 \\
\hline 71 & Maíz & SO. Buenos Aires & 7 & 55 & $168 / 56$ & ene-03 & dic-16 \\
\hline 72 & Maíz & SO. Buenos Aires & 7 & 70 & $168 / 56$ & ene-03 & dic-16 \\
\hline 81 & Trigo & N. Buenos Aires - S. Santa Fe & 8 & 35 & $168 / 56$ & ene-03 & $\operatorname{dic}-16$ \\
\hline 82 & Trigo & N. Buenos Aires - S. Santa Fe & 8 & 65 & $168 / 56$ & ene-03 & $\operatorname{dic}-16$ \\
\hline 91 & Trigo & SE. Buenos Aires & 9 & 60 & $168 / 56$ & ene-03 & $\operatorname{dic}-16$ \\
\hline 92 & Trigo & SE. Buenos Aires & 9 & 65 & $168 / 56$ & ene-03 & dic-16 \\
\hline 101 & Trigo & SE. Buenos Aires & 10 & 65 & $168 / 56$ & ene-03 & dic-16 \\
\hline 102 & Trigo & SE. Buenos Aires & 10 & 50 & $168 / 56$ & ene-03 & dic-16 \\
\hline 111 & Trigo & SO. Buenos Aires & 11 & 26 & $168 / 56$ & ene-03 & dic-16 \\
\hline 112 & Trigo & SO. Buenos Aires & 11 & 36 & $168 / 56$ & ene-03 & $\operatorname{dic}-16$ \\
\hline 121 & Trigo & SE. Córdoba & 12 & 25 & $168 / 56$ & ene-03 & dic-16 \\
\hline 122 & Trigo & SE. Córdoba & 12 & 35 & $168 / 56$ & ene-03 & $\operatorname{dic}-16$ \\
\hline 131 & Soja & N. Buenos Aires & 13 & 36 & $168 / 56$ & ene-03 & dic-16 \\
\hline 132 & Soja & N. Buenos Aires & 13 & 38 & $168 / 56$ & ene-03 & $\operatorname{dic}-16$ \\
\hline 141 & Soja & 0. Buenos Aires & 14 & 36 & $168 / 56$ & ene-03 & dic-16 \\
\hline 142 & Soja & 0. Buenos Aires & 14 & 38 & $168 / 56$ & ene-03 & dic-16 \\
\hline 151 & Soja & SE. Buenos Aires & 15 & 26 & $168 / 56$ & ene-03 & dic-16 \\
\hline 152 & Soja & SE. Buenos Aires & 15 & 32 & $168 / 56$ & ene-03 & dic-16 \\
\hline
\end{tabular}

Notas: ${ }^{a}$ Combinación de insumos y labores. Los números son indicativos para diferenciar las técnicas, ${ }^{b}$ Quintales producidos por hectárea.

Fuente: elaboración propia con base en la revista Márgenes Agropecuarios. 
Pablo Wahren

Cuadro 2. Variables explicativas y a explicar

\begin{tabular}{lll}
\hline Variable & Fuente & Unidad \\
\hline Variables explicativas & & \\
\hline Tipo de cambio nominal & BCRA & Peso arg. \\
Salario medio & Mtro. de Trabajo, Empleo y Seguridad Social & Peso arg. \\
Salario producción de semillas s.e. & Mtro. de Trabajo, Empleo y Seguridad Social & Peso arg. \\
Salario producción de agroquímicos s.e. & Mtro. de Trabajo, Empleo y Seguridad Social & Peso arg. \\
Salario peón s.e. & CREA & Peso arg. \\
Gasoil & CREA & Peso arg. \\
Precio soja & FMI & Dólar \\
Precio maíz & FMI & Dólar \\
Precio trigo & FMI & Dólar \\
Precio petróleo & FMI & Dólar \\
Derechos de exportación soja & Bolsa de Cereales de Rosario & Tasa \\
Derechos de exportación maíz & Bolsa de Cereales de Rosario & Tasa \\
Derechos de exportación trigo & Bolsa de Cereales de Rosario & Tasa \\
Cambio técnico soja & Márgenes Agropecuarios & Dummy \\
Cambio técnico maíz & Márgenes Agropecuarios & Dummy \\
Cambio técnico soja & Márgenes Agropecuarios & Dummy \\
\hline Variables dependientes & Márgenes Agropecuarios & Dólar \\
\hline Labranza & Márgenes Agropecuarios & Dólar \\
Semillas & Márgenes Agropecuarios & Dólar \\
Agroquímicos & Márgenes Agropecuarios & Dólar \\
Gastos de comercialización & Márgenes Agropecuarios & Cosecha \\
\hline
\end{tabular}

Fuente: elaboración propia.

Para realizar la econometría de panel se optó por un modelo de efectos aleatorios (Random Effects o RE). El mismo asume que existen diferencias entre regiones (dada la técnica productiva imperante en cada una) y que el efecto específico de la técnica es una variable aleatoria no correlacionada con las co- 
variables. RE permite estimar un intercepto diferente para cada unidad transversal, en este caso para cada técnica productiva. El modelo se expresa como:

$$
\operatorname{Costo}_{i t}=\alpha_{i}+\beta_{1} X_{1 i t}+e_{i t}
$$

Donde $\alpha_{i}=\alpha+u_{i}$.

Esto quiere decir que el intercepto pasa a estar compuesto por una variable aleatoria con media en $\alpha$ y una desviación aleatoria $u_{i}$. Sustituyendo $\alpha_{i}=\alpha+u_{i}$ en (1), se obtiene:

$$
\text { Costo }_{i t}=\alpha+\beta_{1} X_{1 i t}+u_{i}+e_{i t}
$$

La elección se fundamenta en que la prueba de Breush y Pagan reportó que existen diferencias entre técnicas, lo que implica que cada unidad transversal tiene un intercepto diferente que debe ser estimado. Si bien los mismos podrían ser estimados también por efectos fijos, el test de Hausman mostró que no existen diferencias significativas entre ambos modelos. En este sentido, conviene utilizar RE, ya que al estimar menos parámetros aumenta los grados de libertad. Resulta razonable que en el modelo desarrollado las variables independientes no se encuentren correlacionadas con el error, dado que las mismas son de carácter nacional por lo que particularidades regionales no deberían afectarlas.

Por su parte, el test de Wooldbridge detectó presencia de autocorrelación en las regresiones planteadas. Asimismo, mediante el test de Wald y el de Pesaran se infiere presencia de heterocedasticidad en algunas de las regresiones y correlación contemporánea en la totalidad de las mismas. Por tales motivos, se complementarán las estimaciones de RE con los estimadores Mínimos Cuadrados Generalizados Factibles (Feasible Generalized Least Squares o FGLS) o Errores Estándar Corregidos para Panel (Panel Corrected Standard Errors o PCSE) (Beck, 2001) a fin de erradicar conjuntamente los tres problemas. Por último, cabe señalar que las series se trabajaron en diferencias para eliminar la estacionariedad detectada por el test Dicky Fuller Aumentado y por el test de Harris-Tsvalis.

\section{Especificación del modelo}

A fin de aproximarse al grado de dolarización de los costos, se estimarán las elasticidades al tipo de cambio de cada uno de los principales costos agríco- 
las. El resultado esperado es que los costos, medidos y expresados en dólares, de los ítems típicos del nuevo modelo productivo (semillas y agroquímicos) tengan una sensibilidad menor. En otras palabras, incrementos del tipo de cambio tienden a dejar inalterado el precio en dólares de este primer grupo. Asimismo, se buscará conocer la sensibilidad de los costos totales al tipo de cambio.

En Argentina, Frenkel (1986) realizó una importante contribución para clarificar los determinantes directos de la inflación desde el enfoque de costos. Desde su punto de vista los precios se pueden descomponer en cuatro componentes: precios flexibles (p. flex), tipo de cambio (e), salarios (w) y precios regulados (p. gob). Cada uno de ellos tiene asignado un coeficiente y en su conjunto permiten explicar la dinámica de los precios generales de la economía. De este análisis se desprende una ecuación del tipo:

$$
p_{t}=\beta_{0} p_{t}^{\text {flex }}+\beta_{1} e_{t}+\beta_{2} w_{t}+\beta_{3} p_{t}^{g o b}
$$

En un trabajo más reciente, Frenkel y Friendheim (2016) desagregaron esta ecuación en más componentes e incorporaron un componente autorregreresivo de los precios, como factor que incorpora la inercia inflacionaria e incorpora otros factores no incluidos. Asimismo, realizaron estimaciones para Argentina a partir de un modelo mensual en primeras diferencias.

Las ecuaciones que describen el comportamiento de los costos agrícolas se guiaron por los criterios propuestos en trabajos precedentes y fueron elaboradas considerando la literatura y las entrevistas en profundidad con actores del sector. $\mathrm{Al}$ igual que en los trabajos mencionados, se incorpora un componente autorregresivo, y las variables se presentan en logaritmos y en primeras diferencias. Cabe aclarar que en el caso de la cosecha se aplicó una media móvil de tres meses, dada su volatilidad.

\section{Labranza}

Para cuantificar el impacto del tipo de cambio sobre el costo de labranza se controlará por el salario de los trabajadores agrarios y por el precio del gasoil. Se incorpora también una dummy para registrar el cambio técnico presentado por la revista y captar las variaciones en los costos derivadas de este efecto.

$$
\begin{aligned}
\text { labranzacultivo }_{i, t}= & \beta_{0}+\beta_{1} \text { labranzacultivo }_{i, t-1}+\beta_{2} e_{t}+\beta_{3} \text { wpeon }_{t} \\
& +\beta_{4} p_{t}^{\text {gasoil }}+\beta_{5} C T_{i}+u_{i t}
\end{aligned}
$$




\section{Semillas}

En el caso de las semillas se controlará por el salario del sector, el precio internacional del cultivo en relación con los últimos tres trimestres y las retenciones. El hecho de tomar el acumulado de los trimestres se debe a lo recabado en las entrevistas, acerca de que la reacción a los precios tiende a demorarse y a comprobarse un mayor coeficiente de determinación al incluir la variable con rezago. Se incluyen las retenciones porque influyen sobre el precio que obtienen los productores locales. Por último, también se incorpora la dummy de cambio técnico.

$$
\begin{aligned}
\text { semillacultivo }_{i, t}= & \beta_{0}+\beta_{1} \text { semillacultivo }_{i, t-1}+\beta_{2} e_{t}+\beta_{3} \text { wsemila }_{t} \\
& +\beta_{4} p_{t}^{\text {cultivosq }}+\beta_{5} \text { retcultivo }_{t}+\beta_{6} C T_{i}+u_{i t}
\end{aligned}
$$

\section{Agroquimicos}

En el caso de los agroquímicos, se controla por el salario del sector, los precios internacionales del cultivo y los precios internacionales del petróleo en relación a tres trimestres atrás. El hecho de incluir el precio de los cultivos se asocia a la mencionada disputa al interior de la cadena. Por su parte, se incorpora el petróleo por ser uno de los principales insumos de producción de diversos agroquímicos y fertilizantes. Por último, se agrega la variable dummy de cambio técnico.

$$
\begin{aligned}
\text { agroqcultivo }_{i, t}= & \beta_{0}+\beta_{1} \text { agroqcultivo }_{i, t-1}+\beta_{2} e_{t}+\beta_{3} \text { wagroq }_{t} \\
& +\beta_{4} p_{t}^{\text {cultivo } 3 q}+\beta_{5} p_{t}^{\text {petro3q }}+\beta_{6} C T_{i}+u_{i t}
\end{aligned}
$$

\section{Cosecha}

En cuanto a la cosecha, las variables de control son el salario, el gasoil, los precios internacionales al momento presente y las retenciones. Dado que el precio tiende a fijarse en relación con el grano recolectado influye el precio presente del cultivo. En este sentido, también se incorporan las retenciones por afectar la evolución del precio local de estos últimos.

$$
\begin{aligned}
\text { cosechacultivo }_{i, t}= & \beta_{0}+\beta_{1} \text { cosechacultivo }_{i, t-1}+\beta_{2} e_{t}+\beta_{3} \text { wpeon }_{t} \\
& +\beta_{4} p_{t}^{\text {gasoil }}+\beta_{5} \text { cultivo }_{t}+\beta_{6} \text { retcultivo }_{t}+u_{i t}
\end{aligned}
$$




\section{Comercialización}

Para los gastos de comercialización se controlará por el salario medio, gasoil, precios internacionales coyunturales y retenciones. Los primeros influyen fundamentalmente en el costo de transporte. Los segundos son relevantes para las otras tareas que componen estos costos como acopio, secado y zarandeo, ya que se valúan a porcentaje del precio de venta del producto.

$$
\begin{aligned}
\text { comercultivo }_{i, t}= & \beta_{0}+\beta_{1} \text { comercultivo }_{i, t-1}+\beta_{2} e_{t}+\beta_{3} \text { wmedio }_{t} \\
& +\beta_{4} p_{t}^{\text {gasoil }}+\beta_{5} p_{t}^{\text {cultivo }}+\beta_{6} \text { retcultivo }_{t}+u_{i t}
\end{aligned}
$$

\section{Costos totales}

Por último, para los costos totales se incluirán todas las variables mencionadas anteriormente.

$$
\begin{aligned}
\text { costocultivo }_{i, t}= & \beta_{0}+\beta_{1} \text { costocultivo }_{i, t-1}+\beta_{2} e_{t}+\beta_{3} \text { wmedio }_{t} \\
& +\beta_{4} p_{t}^{\text {gasoil }}+\beta_{5} p_{t}^{\text {cultivo }}++\beta_{6} p_{t}^{\text {petro3q }}+\beta_{7} p_{t}^{\text {cultivo } 3 q} \\
& +\beta_{8} \text { retcultivo }_{t}+\beta_{9} C T_{i}+u_{i t}
\end{aligned}
$$

\section{RESULTADOS}

Como se mencionó, se presentan las estimaciones por RE, FGLS y PCSE para cada uno de los ítems de costos y para los costos totales. Al analizar los resultados de la labranza se registra un impacto negativo significativo y de magnitud del tipo de cambio. En el caso del maíz, el coeficiente oscila entre -0.54 y -0.59 según el modelo. En el caso de la soja el impacto registrado es mayor, entre -0.69 y -0.70 . Mientras que el trigo presenta la mayor amplitud entre los modelos, registrando un coeficiente de -0.42 por FGLS y de -0.65 con los restantes métodos. Los signos de las demás variables son los esperados. El salario y el gasoil, principales insumos de esta tarea, presentan coeficientes significativos y positivos. La única excepción es el salario en el caso de la soja al estimar por efectos aleatorios, donde el resultado es positivo, pero no significativo. Por su parte, el componente autorregresivo y la dummy de cambio técnico no resultan significativas salvo en la estimación FGLS del maíz. 
En el caso de las semillas, el efecto del tipo de cambio es mucho menor en línea con lo esperado. Puntualmente en las semillas soja y el trigo, el impacto de sus variaciones no es significativo. En el caso del maíz, en las estimaciones RE y FGLS, el coeficiente sí es significativo y negativo, pero en una magnitud muy inferior a lo registrado en labranza ( -0.15 en RE y -0.16 en FLGS). Los precios internacionales resultan significativos en todas las estimaciones, con excepción del maíz bajo PCSE. En todos los casos el signo es positivo. Como ya se mencionó, los precios internacionales impactan en los costos de las semillas por dos cuestiones: la primera, por ser el grano insumo de producción, y la segunda, por una tendencia de los actores del rubro a ser parte de los mayores ingresos derivados de precios internacionales más elevados. Las retenciones, en los casos donde son significativas, presentan signo negativo. Lo cual iría en línea con el impacto negativo sobre el precio de venta de los cultivos que esta medida genera. El salario de los trabajadores del sector sólo presenta significatividad en las estimaciones de maíz donde reporta signo positivo. El componente autorregresivo es significativo en la mayoría de las estimaciones y positivo. El cambio técnico tiene signo negativo en los dos casos donde presenta significatividad.

En el caso de los agroquímicos (incluidos fertilizantes) se observa una dinámica similar a la del sector semillas, con el que comparten el carácter industrial, un alto grado de transabilidad y en muchos casos el protagonismo de los mismos actores. El tipo de cambio no resulta significativo, con la única excepción del maíz estimado por RE. El precio de cada cultivo resulta significativo y positivo, lo cual en este caso parece asociarse más a la intención de los actores de aprovechar coyunturas favorables en los precios internacionales aumentándolos. En todos los casos, el coeficiente supera el 0.4. El precio del petróleo, insumo de producción relevante de diversos agroquímicos y fertilizantes, también resulta significativo y positivo. El salario del sector presenta resultados dispares. Siendo significativo y positivo en el caso del maíz bajo las estimaciones RE y FGLS; no significativo en maíz y significativo, pero negativo, en las estimaciones RE y PCSE de trigo. El componente autorregresivo resulta significativo y positivo, mientras que el cambio técnico es no significativo salvo en el caso del trigo bajo RE y PCSE, donde es significativo y levemente positivo.

En la cosecha el tipo de cambio vuelve a ser negativo para el maíz, bajo RE y FGLS, y en todas las estimaciones del trigo. En soja resulta no significativo. En cualquier caso, la magnitud de los coeficientes fue considerablemente inferior a la del caso de la labranza (en torno a -0.08 en maíz y de -0.16 en trigo). Los salarios resultaron significativos y positivos, salvo en las estimaciones PCSE 
para soja y trigo. En todas las estimaciones el coeficiente fue inferior al 0.10. En cambio, el gasoil resultó no significativo. La poca relevancia de los principales costos de esta tarea se asocia a la forma en que se determina el precio de la cosecha, que al ser realizada por un contratista se fija principalmente en función de los precios del producto levantado, con una cota inferior asociada a un mínimo de ingresos asegurado. En línea con este razonamiento, los precios de cada cultivo presentan coeficientes significativos y positivos. Por su parte, las retenciones, en los casos donde son significativas, presentan signos negativos. Por último, el coeficiente autorregresivo resulta significativo y positivo.

Tal como se esperaba, en los gastos de comercialización el tipo de cambio cumple un rol importante. El impacto de sus variaciones es significativo y negativo. En el caso del maíz, los coeficientes se ubican en línea a -0.57 , en soja a -0.61 y en trigo a -0.50 . Esta dinámica se asocia a que el principal gasto de comercialización es el transporte, cuyos costos operativos se encuentran poco dolarizados al requerir mano de obra y combustibles. En efecto, tanto el salario como el gasoil presentan coeficientes significativos y positivos. El precio de cada cultivo también tiene efectos positivos, lo que se asocia a otros tipos de costos de comercialización como acopio, secada o zarandeo que se realizan a porcentaje de lo entregado. El componente autorregresivo no resulta significativo.

Para finalizar, en los costos totales el tipo de cambio resulta significativo y negativo, con la única excepción de la estimación de trigo realizada por PCSE. Como era de esperar, su impacto es inferior al registrado en labranza y cosecha, pero superior al obtenido en los otros ítems. En promedio se ubica en -0.23, en soja en -0.27 y en trigo en -0.31 . La menor elasticidad registrada en el maíz puede deberse al mayor peso que detenta el paquete tecnológico en este cultivo, el cual se asocia al mayor ritmo de innovaciones, mayores barreras a la entrada y dificultad para la imitación que se presentan las semillas de este cultivo (Sztulwark, 2012). Los componentes locales de los costos, como salarios y gasoil, presentan signo positivo, aunque su significatividad difiere según cada estimación. Los precios internacionales con rezago, tanto del petróleo como de cultivos, son positivos y significativos. No así los precios internacionales del cultivo contemporáneo, donde sólo el maíz se registra significatividad. Las retenciones presentan signo negativo, pero sólo son significativas en las estimaciones RE y FGLS de maíz. En la estimación de trigo por FGLS se registra un resultado no esperado, el signo significativo y positivo de las retenciones. El componente autorregresivo es positivo y significativo en la mayor parte de las estimaciones. Por último, el cambio técnico resultó no significativo. 


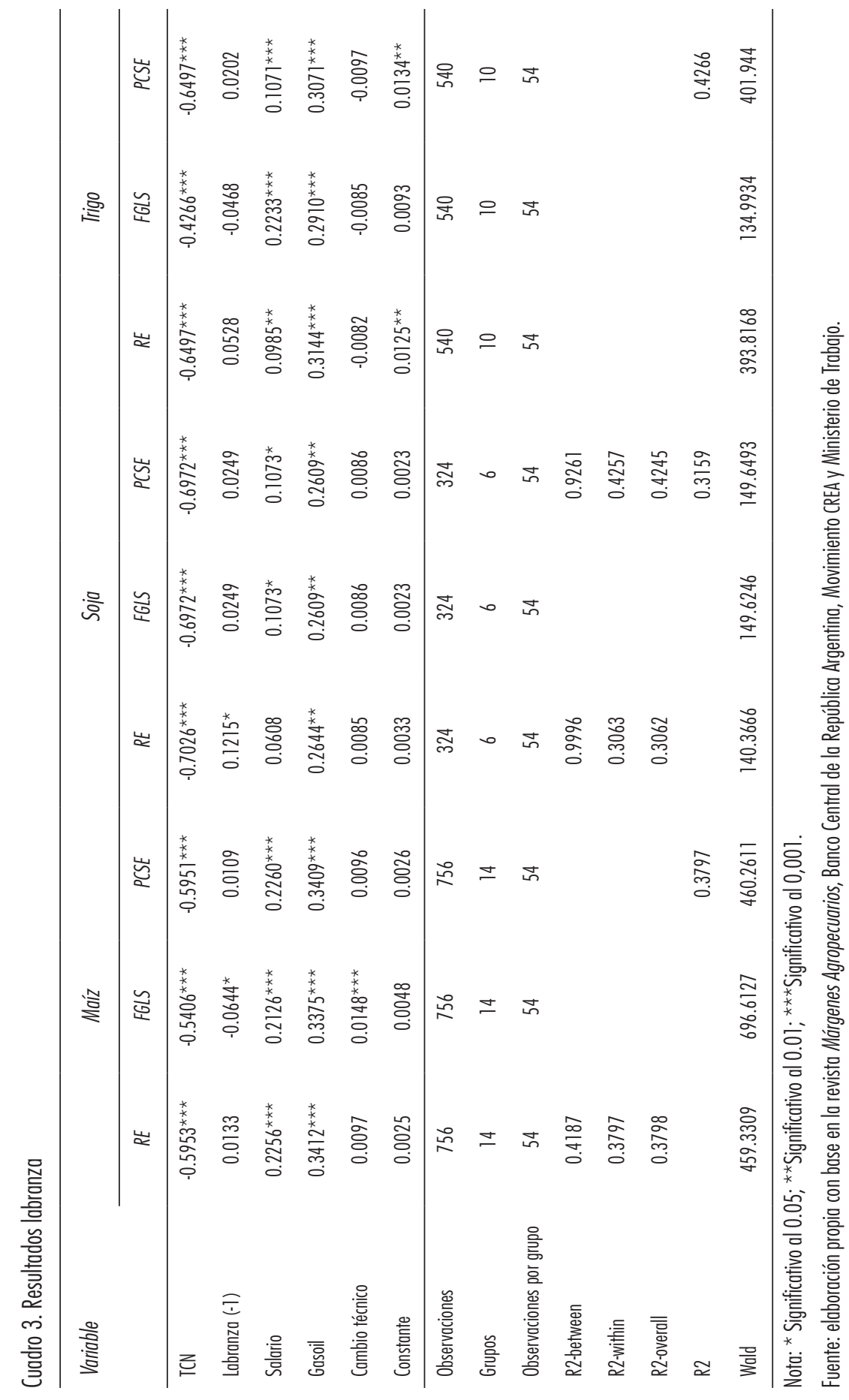




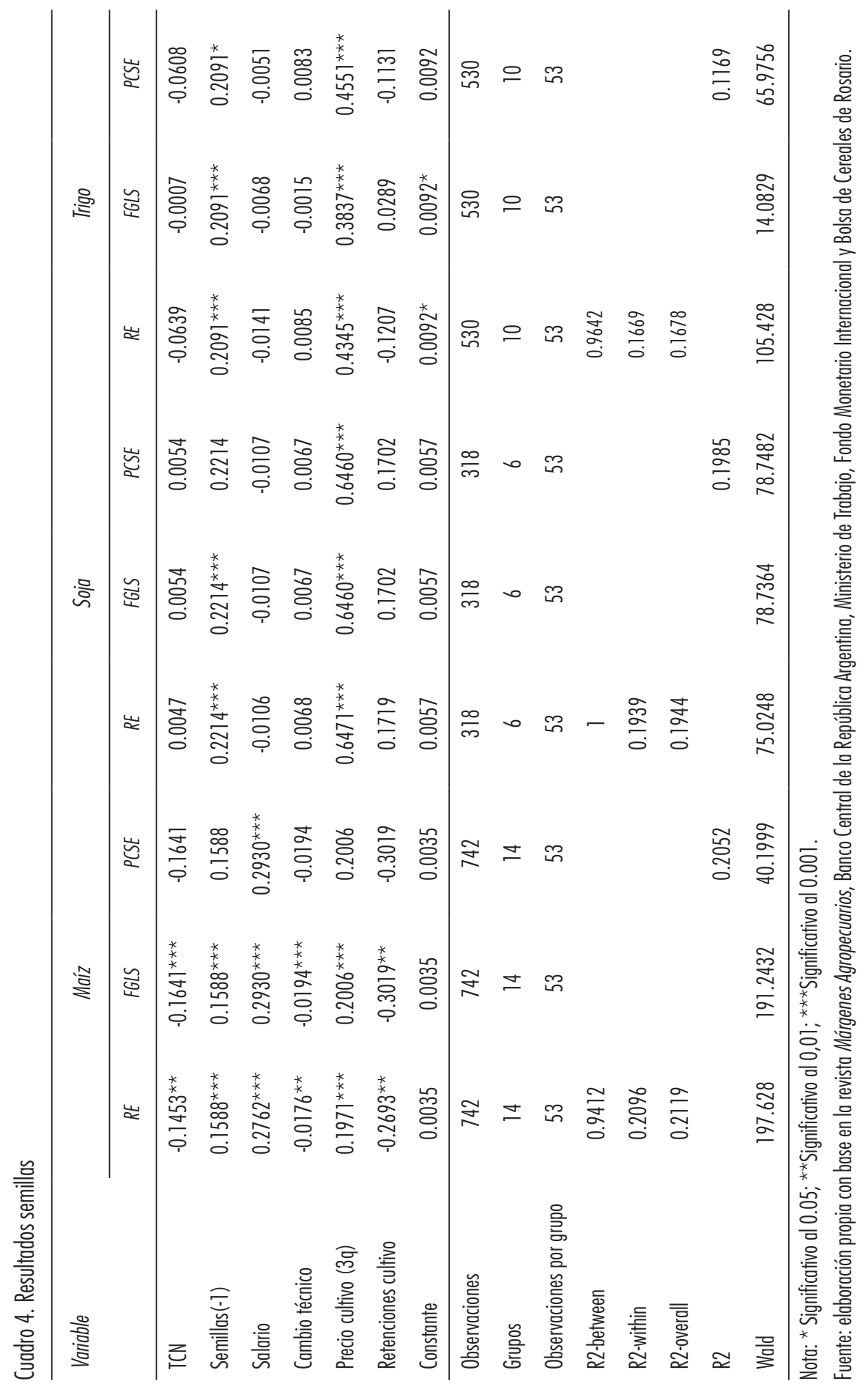




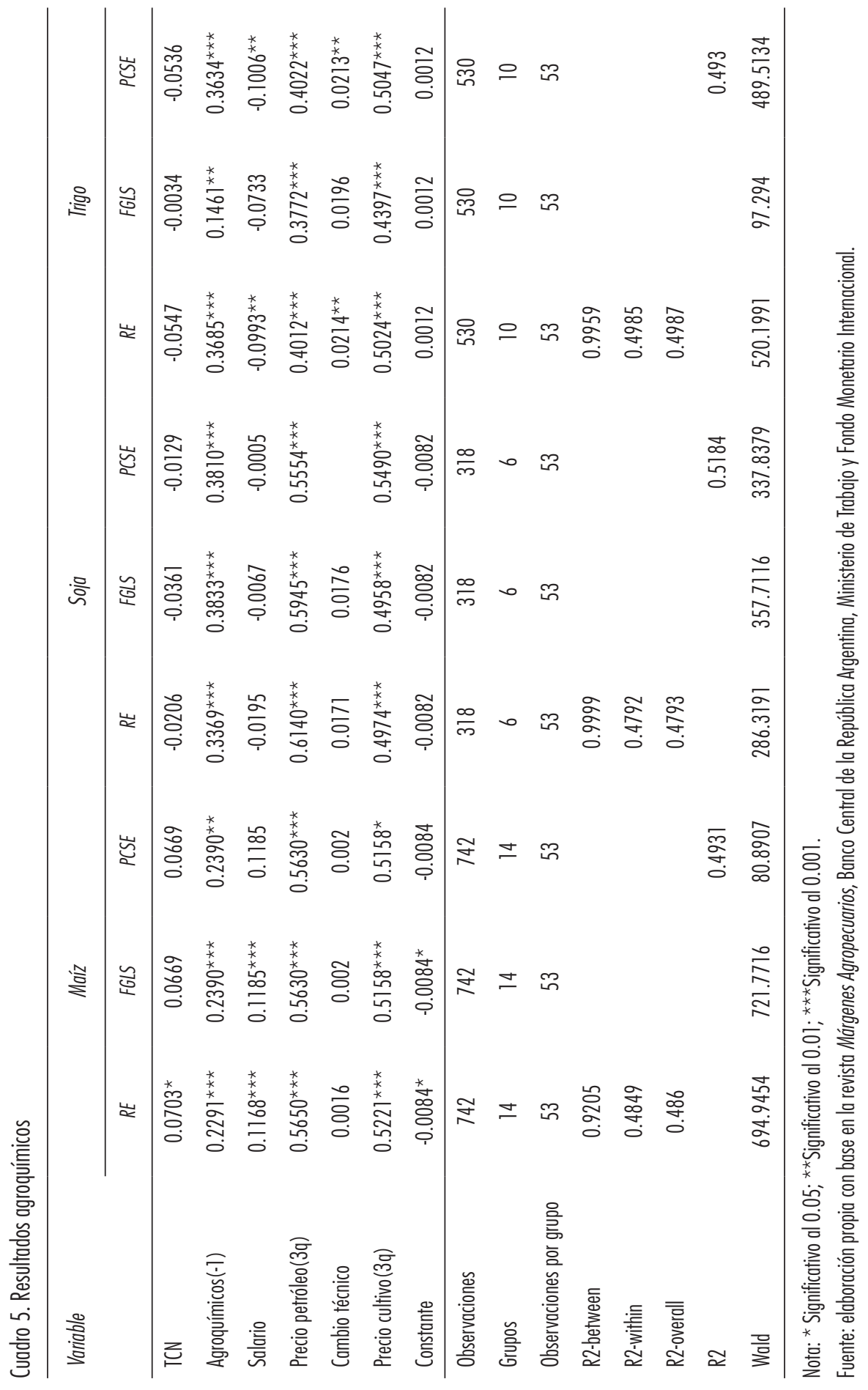




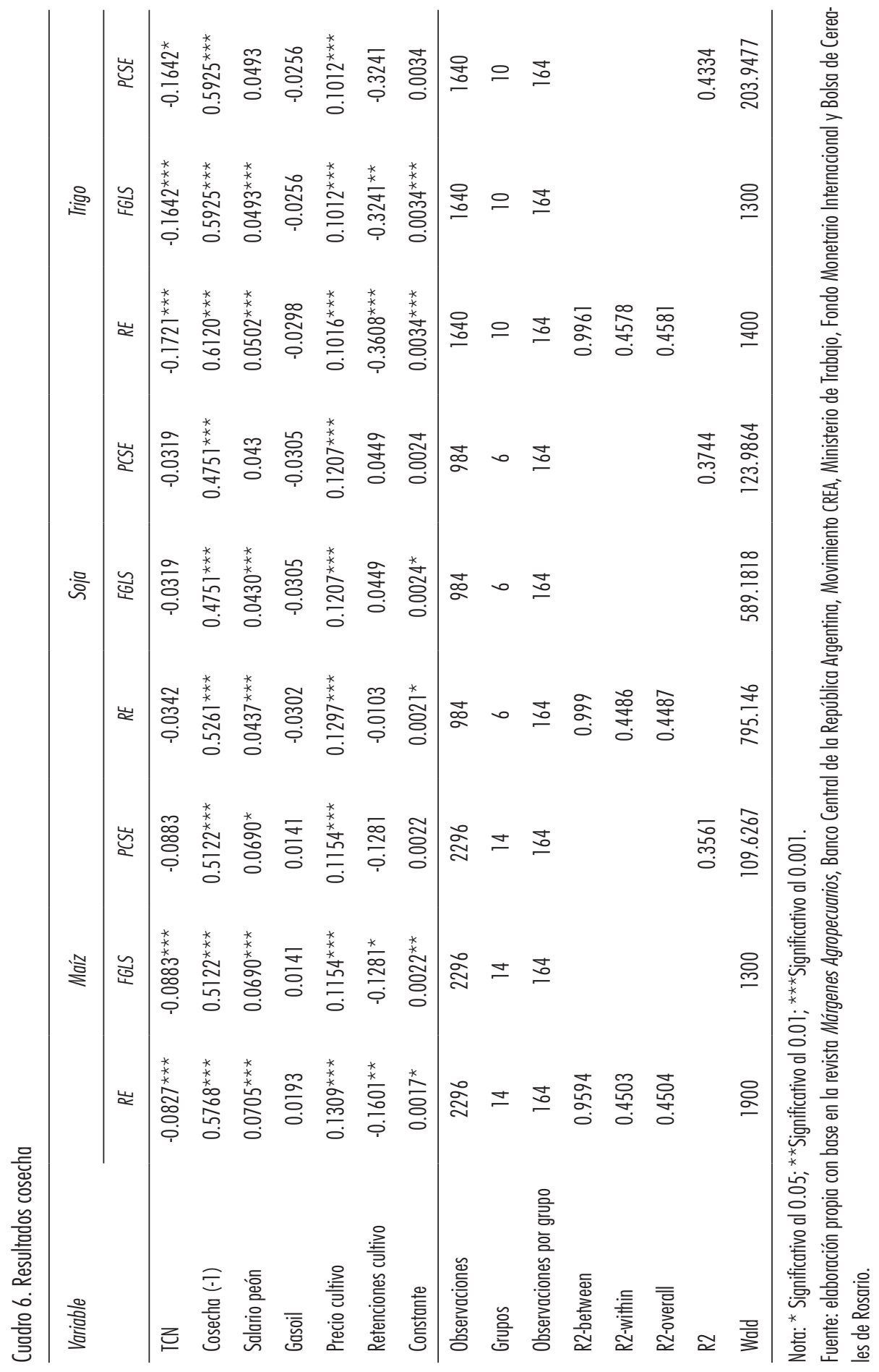




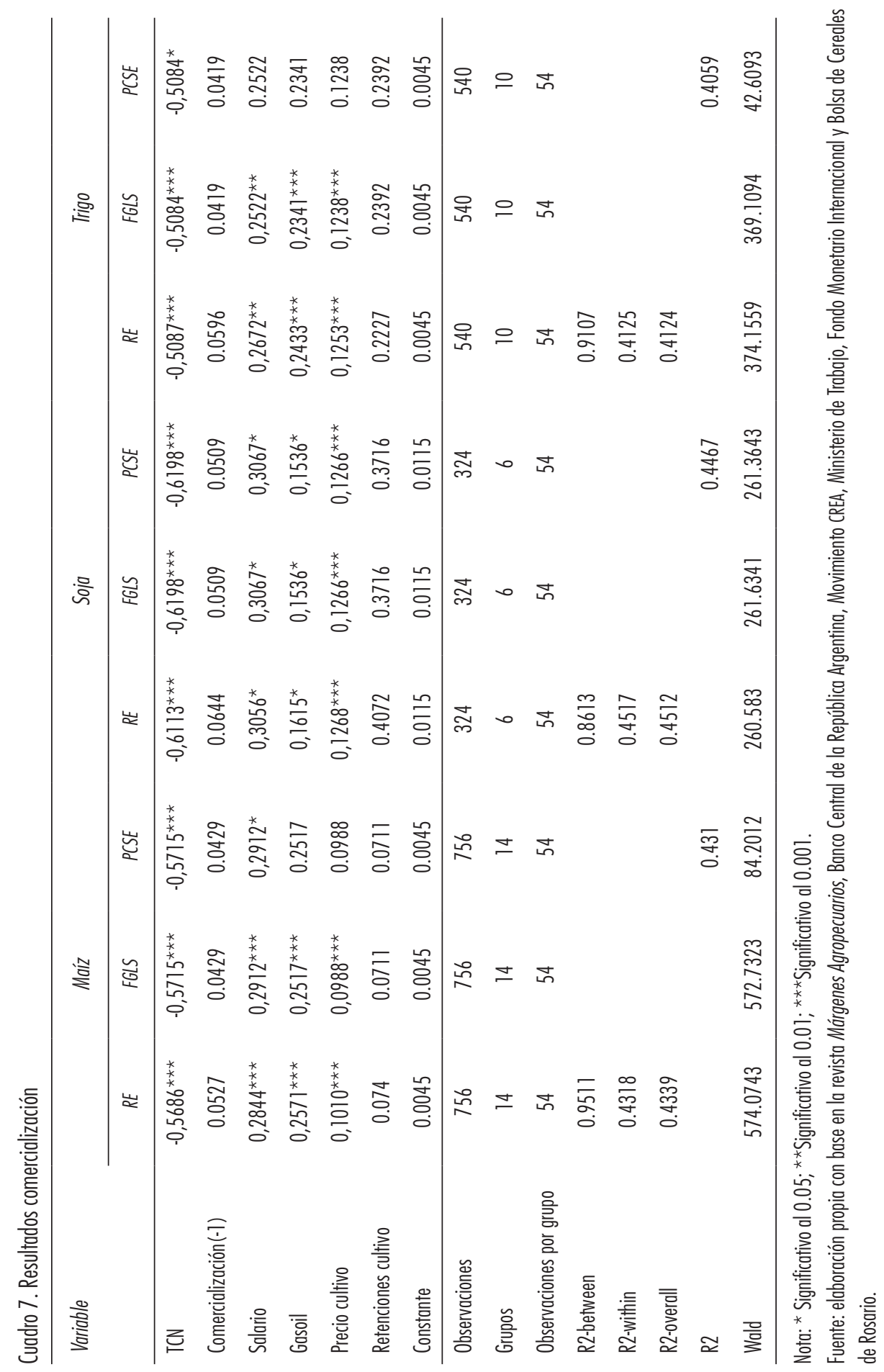




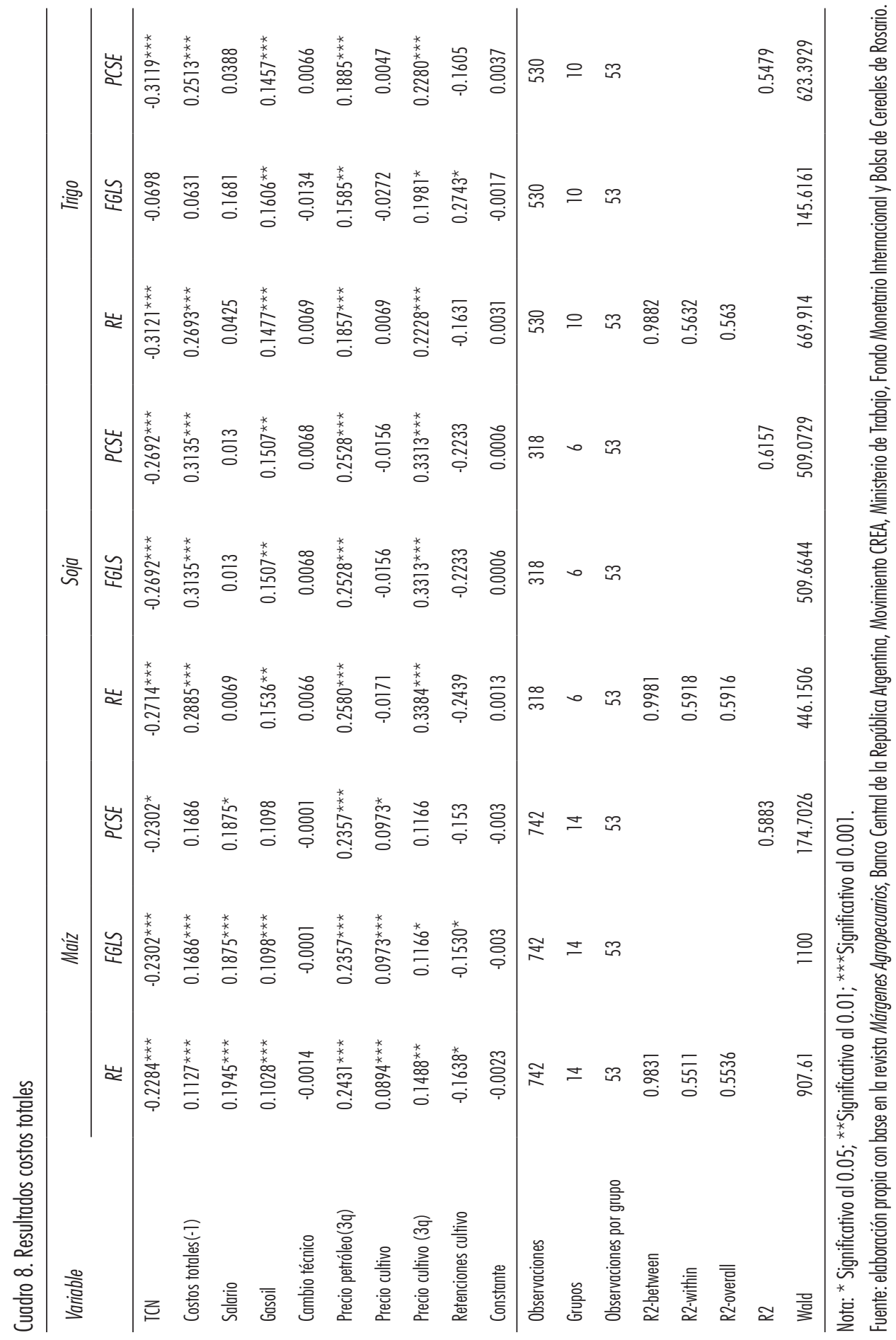


En resumen, en las distintas estimaciones se aprecia cómo en cada cultivo se repite la misma relación entre costos y tipo de cambio:

- Un impacto significativo y muy negativo en labranza y comercialización.

- Un impacto no significativo, o levemente negativo en los casos donde se registra significatividad, en semillas y agroquímicos.

- Un impacto negativo en cosecha, pero más leve y con un menor grado de significatividad.

Cuadro 9. Impacto del tipo de cambio por tipo de costo

\begin{tabular}{|c|c|c|c|c|c|c|c|}
\hline Cultivo & Estimación & Labranza & Semillas & Agroquímicos & Cosecha & Comercialización & $\begin{array}{l}\text { Costos } \\
\text { totales }\end{array}$ \\
\hline \multirow[t]{3}{*}{ Maíz } & RE & $-0.5953^{\star \star \star}$ & $-0.1453^{\star \star}$ & $0.0703^{*}$ & $-0.0827^{\star \star \star}$ & $-0.5686^{\star \star \star}$ & $-0.2284^{\star \star \star}$ \\
\hline & FGLS & $-0.5406^{\star \star \star}$ & $-0.1641^{\star \star \star}$ & 0.0669 & $-0.0883^{\star \star \star}$ & $-0.5715^{\star \star \star}$ & $-0.2302^{\star \star \star}$ \\
\hline & PCSE & $-0.5951^{\star \star \star}$ & -0.1641 & 0.0669 & -0.0883 & $-0.5715^{\star \star \star}$ & $-0.2302^{*}$ \\
\hline \multirow[t]{3}{*}{ Soja } & RE & $-0.7026^{\star \star \star}$ & 0.0047 & -0.0206 & -0.0342 & $-0.6113^{\star \star \star}$ & $-0.2714^{\star \star \star}$ \\
\hline & FGLS & $-0.6972^{\star \star \star}$ & 0.0054 & -0.0361 & -0.0319 & $-0.6198^{\star \star \star}$ & $-0.2692^{\star \star \star}$ \\
\hline & PCSE & $-0.6972^{\star \star \star}$ & 0.0054 & -0.0129 & -0.0319 & $-0.6198^{\star \star \star}$ & $-0.2692^{\star \star \star}$ \\
\hline \multirow[t]{3}{*}{ Trigo } & RE & $-0.6497^{\star \star \star}$ & -0.0639 & -0.0547 & $-0.1721^{\star \star \star}$ & $-0.5087^{\star \star \star}$ & $-0.3121^{\star \star \star}$ \\
\hline & FGLS & $-0.4266^{\star \star \star}$ & -0.0007 & -0.0034 & $-0.1642^{\star \star \star}$ & $-0.5084^{\star \star \star}$ & -0.0698 \\
\hline & PCSE & $-0.6497^{\star \star \star}$ & -0.0608 & -0.0536 & $-0.1642^{\star}$ & $-0.5084^{\star}$ & $-0.3119^{\star \star \star}$ \\
\hline
\end{tabular}

Nota: * Significativo al $0.05 ;$; Significativo al $0.01 ; * \star *$ Significativo al 0.001 .

Fuente: elaboración propia con base en la revista Márgenes Agropecuarios, Banco Central de la República Argentina, Movimiento CREA, Ministerio de Trabajo, Fondo Monetario Internacional y Bolsa de Cereales de Rosario.

\section{CONCLUSIONES}

El presente artículo busca conocer el grado de dolarización de los costos agrícolas. De las estimaciones realizadas, se concluye que el impacto del tipo de cambio sobre los costos medidos en dólares oscila entre - 0.22 y - 0.31 según el cultivo. Es decir, un incremento del tipo de cambio de $1 \%$ genera reducciones de los costos en dólares en la mencionada cuantía. Tres aspectos centrales en lo que se refiere al objeto de estudio se deducen del análisis precedente: 
- Las depreciaciones cambiarias impactan en los ingresos que recibe el sector en moneda doméstica, pero también en sus costos, ya que sino la reducción de costos en dólares debería acompañar a la depreciación. Este efecto debe ser tomado en cuenta a la hora de analizar cómo evoluciona la rentabilidad.

- Según las estimaciones, los márgenes brutos promedio de los tres cultivos seleccionados se incrementan con las depreciaciones del tipo de cambio, tanto en dólares como en moneda local. Sin embargo, este aumento se ve suavizado por la lógica dolarizada de los insumos agrícolas.

- Un aspecto que explica la baja sensibilidad de los costos agrícolas (en dólares) a variaciones del tipo de cambio es el nuevo modelo productivo, por haber elevado el grado de transabilidad de insumos y establecido nuevas reglas de fijación de precios. Se observa que los aspectos claves del modelo (semillas y agroquímicos) son los más dolarizados.

Los aspectos mencionados en líneas precedentes permiten plantear algunas reflexiones vinculadas con las discusiones sobre política económica. Si como se desprende de la investigación, las depreciaciones del tipo de cambio impactan en los ingresos que recibe el sector, pero también en sus costos, este efecto debe ser considerado a la hora de analizar cómo evoluciona la rentabilidad. Asimismo, un aspecto adicional para tener en cuenta es la dinámica de los ingresos según cultivo. Pues, mientras que en la soja la traslación del tipo de cambio a los precios es inmediata - por ser un producto que se exporta casi en su totalidad-, en maíz y trigo los molinos y feed-lots median entre el precio establecido en la bolsa de cereales y el efectivamente pagado por las ventas el mercado interno.

Es preciso remarcar que los derechos de exportación constituyen una herramienta sumamente relevante para desacoplar precios, y que otros impuestos sobre el sector difícilmente puedan tener el mismo efecto. Sin embargo, de lo expuesto se desprenden dos aspectos que complejizarían el diseño de devaluaciones compensadas:

1) El efecto negativo sobre el excedente agrícola de las retenciones no es equivalente a devaluaciones de igual magnitud, lo que derivaría en cambios distributivos con implicancias políticas.

2) En caso de que la totalidad de la renta agrícola ya sea captada por los derechos de exportación, un incremento por encima de ese nivel podría generar dificultades en la propia reproducción de la actividad. 
Como consecuencia, un esquema de devaluaciones compensadas que no afecte la rentabilidad del sector debería basarse en retenciones móviles que sigan variables de ingresos y de costos. Sin embargo, implicaría asumir el carácter regresivo de las devaluaciones compensadas, ya que los alimentos sufrirían incrementos de precios en función de los aumentos de los costos. Lo que se observa a partir de la dolarización de los costos agrícolas es una imposibilidad de implantar esquemas de tipo de cambio alto sin alterar la distribución del ingreso. En este sentido, los hacedores de política económica al elegir estos regímenes cambiarios deberán decidir entre afectar la rentabilidad de la cadena agrícola, disminuir el salario real, o distribuir ingreso desde otro sector económico o eslabón de la cadena. Por lo tanto, así como los efectos de diversas políticas económicas están siendo reexaminados a nivel internacional como consecuencia de la globalización, los efectos que ésta tuvo sobre el sector agrario imponen nuevos desafíos para el diseño de políticas económicas propias de la periferia.

\section{BIBLIOGRAFÍA}

Abeles, M. y Panigo, D. (2015), "Política antiinflacionaria en un contexto de creciente volatilidad en los precios internacionales de productos básicos", en A. Bárcena, A. Prado y M. Abeles (eds.), Estructura productiva y politica macroeconómica. Enfoques heterodoxos desde América Latina, Chile, CEPAL.

Beck, N. (2001), "Time-series-cross-section data: What have we learned in the past few years?”, Annual Review of Political Science, vol. 4, núm. 27193, DOI <https://doi.org/10.1146/annurev.polisci.4.1.271>

Bisang, R. (2007), "El desarrollo agropecuario en las últimas décadas: ¿volver a creer?, en B. Kosacoff (ed.), Crisis, recuperación y nuevos dilemas. La economia argentina, 2002-2007, Chile, CEPAL.

Bisang, R., Anlló, G. y Campi, M. (2008), "Una revolución (no tan) silenciosa. Claves para repensar el agro en Argentina”, Desarrollo Económico, vol. 48, núm. 190/191, Argentina, IDEs, julio-diciembre.

, Salvatierra, G. y Anlló, G. (eds.) (2010), “Cambios estructurales en las actividades agropecuarias: de lo primario a las cadenas globales de valor", Chile, CEPAL: Colección Documentos de Proyectos.

Bolsa de Cereales (2018), "Informe cierre de campaña Soja 2017/18”, Departamento de Estimaciones Agricolas, Argentina.

Bresser-Pereira, L. (2007), "El nuevo desarrollismo y la ortodoxia convencional”, Economía UNAM, vol. 4, núm. 10, México, UnAM. 
CREA (2018), "Informe Microeconómico No 58". Recuperado de <https:// www.crea.org.ar/informe-microeconomico-no-58/>

Crespo, E. y Lazzarini, A. (2012), "Un modelo para interpretar las estructuras productivas desequilibradas", Conferencia ESHET Argentina paises de centro y periferia: lecciones de la historia económica y de la historia del pensamiento económico, Buenos Aires, noviembre.

Diamand, M. (1972), "La estructura productiva desequilibrada argentina y el tipo de cambio", Desarrollo Económico, vol. 12, núm. 45, Argentina, IDES.

Diaz Alejandro, C. (1963), "A note on the impact of devaluation and the redistributive effect", Journal of Political Economy, vol. 71, núm. 6, DOI $<$ https://doi.org/10.1086/258816>

Dvoskin, A. y Feldman, G. (2015), "Política cambiaria, distribución del ingreso y estructura productiva”, en A. Bárcena, A. Prado y M. Abeles (eds.), Estructura productiva y politica macroeconómica. Enfoques heterodoxos desde América Latina, Chile, CEPAL.

Fiorito, A., Guaita, N. y Guaita, S. (2015), "Neodesarrollismo y el tipo de cambio competitivo", Cuadernos de Economía, vol. 34, núm. 64, DOI <http://dx.doi.org/10.15446/cuad.econ.v34n64.47385>

Frenkel, R. (1986), "Salarios e inflación en América Latina. Resultados de investigaciones recientes en la Argentina, Brasil, Colombia, Costa Rica y Chile”, Desarrollo Económico, vol. 25, núm. 100, Argentina, IDES.

Frenkel, R. y Ros, J. (2006), "Unemployment and the real exchange rate in Latin America", World Development, vol. 34, núm. 4, Dor <https://doi. org/10.1016/j.worlddev.2005.09.007>

y Friedheim, D. (2016), "La inflación en Argentina en los años 2000", Workshop Central Banks in Latin America: in Search for Stability and Development, Lima, 12 y 13 de mayo.

Gereffi, G. y Sturgeon, T. (2013), "Global value chain-oriented industrial policy: The role of emerging economies", en D. Elms y P. Low (eds.), Global Value Chains in a changing World, Suiza, wTo publications.

Gras, C. y Hernández, V. (2016), Radiografía del nuevo campo argentino. Del terrateniente al empresario transnacional, Argentina, Siglo XXI.

Humphrey, J. y Memedovic, O. (2006), "Global value chains in the agrifood sector", Documento de Trabajo UNIDO. Recuperado de <https://www. unido.org/sites/default/files/2009-05/Global_value_chains_in_the_agrifood_sector_0.pdf>

Instituto Nacional de Estadísticas y Censos (INDEC) (2020), Censo Nacional Agricola. Recuperado de <https://www.indec.gob.ar/ftp/cuadros/publicaciones/cna2018_resultados_preliminares_agricultura.pdf> 
Kicillof, A. y Nahón, C. (2007), "El retorno de la inflación a la Argentina: un problema de salarios y ganancias", Anuario EDI, núm. 3, Argentina, Publicación de los Economistas de Izquierda.

Kosacoff, B. y López, A. (2008), "América Latina y las Cadenas Globales de Valor: debilidades y potencialidades", Globalización, Competitividad y Gobernabilidad, vol. 2, núm. 1, DOI <https://doi.org/10.3232/GCG.2008. V2.N1.01>

Lee, J., Gereffi, G. y Beauvais, J. (2012), "Global value chains and agrifood standards: Challenges and possibilities for smallholders in developing countries", Proceedings of the National Academy of Sciences, vol. 109, núm. 31, DoI <https://doi.org/10.1073/pnas.0913714108>

Lódola, A. (2008), "Contratistas, cambios tecnológicos y organizacionales en el agro argentino", Colección Documentos de Proyectos, núm. 176, Argentina, CEPAL.

López, G. (2008), "Una reconsideración de las perspectivas económicas de México", Economia e Sociedade, núm. 17, DOI < http://dx.doi.org/10.1590/ S0104-06182008000400008>

López, J. y Pacheco, P. (2002), "Políticas de empleo en economías semi-industrializadas", Revista de Economía Política, vol. 22, núm. 2, Brasil, Centro de Economía Política.

Makler, C. A. (2008), "Los discursos de las organizaciones corporativas ruralistas ante los derechos de exportación (1958-62 y 2002-06): materiales para su estudio", Programa Interuniversitario de Historia Politica. Recuperado de <http://historiapolitica.com/datos/biblioteca/agro_makler.pdf> Ministerio de Agroindustria (2016), Mercado de semillas para la siembra de granos en Argentina. Recuperado de <https://www.agroindustria.gob.ar/ new/0-0/programas/dma/granos/Informe\%20Semillas_Agosto\%202016. pdf>

oecd (2018), OECD Economic Outlook, vol. 2018, núm. 1, París, oecD.

Pengue, W. A. (2005), Agricultura industrial y transnacionalización en América Latina: ¿la transgénesis de un continente?, México, Programa de las Naciones Unidas para el Medio Ambiente.

Pierri, J. (2017), "Costos y márgenes en la producción de soja y trigo 19982011: un análisis crítico del concepto de agronegocio", en J. Pierri y R. Bisang (comp.), Problemas actuales y perspectivas futuras de la producción y comercialización de granos, Argentina, Universidad de Buenos Aires.

Razmi, A., Rapetti, M. y Skott, P. (2012), "The real exchange rate and economic development", Structural Change and Economic Dynamics, vol. 23, núm. 2, DOI <https://doi.org/10.1016/j.strueco.2012.01.002> 
Rodrick, D. (2007), "The real exchange rate and economic growth: theory and evidence", Brooking Papers on Economic Activity, vol. 39, núm. 2, Estados Unidos, Brookings Institution Press.

Sztulwark, S. (2012), Renta de innovación en cadenas globales de producción: el caso de las semillas transgénicas en Argentina, Argentina, Universidad Nacional de General Sarmiento.

Teubal, M. (2001), "Globalización y nueva ruralidad en América Latina", en N. Giarraca (comp.), Una nueva ruralidad en América Latina, 45-65, Argentina, CLACSO.

Trigo, E. (2005), "Consecuencias económicas de la transformación agrícola", Ciencia hoy, núm. 87, Argentina, Asociación Civil Ciencia Hoy.

Villulla, J., y Chen, Y. (2015), "Costos y tarifas de los contratistas de cosecha en la agricultura pampeana, 1991-2014", Revista Interdisciplinaria de Estudios Agrarios, núm. 42, Argentina, Centro Interdisciplinario de Estudios Agrarios.

Wahren, P. (2019), Determinantes de los costos en el nuevo agro argentino: aportes para la discusión de politica económica desde un análisis sectorial (Tesis de Maestría), Universidad Nacional de San Martín, Argentina.

Wooldbridge, J. (2002), Econometric analysis of cross section and panel data, Estados Unidos, MIT Press. 